\title{
Theoretical and experimental analysis of the process of discretization of cotton fiber in $\mathrm{OE}$ spinning
}

\author{
Kamol Akhmedov ${ }^{1 *}$, Saypila Matismailov ${ }^{1}$, Botir Mardonov ${ }^{1}$, and Alisher Yuldashev ${ }^{1}$ \\ ${ }^{1}$ Tashkent Institute of Textile and Light Industry, 100100 Tashkent, Uzbekistan
}

\begin{abstract}
This article examines the influence of the parameters of the coating teeth of the discrete drum on the quality of the OE yarn. Based on the analysis of the conditions for retention of fibers by the covering teeth of the discrete drum, equations were obtained to determine the angle of inclination of the teeth. To determine the effect of the coating parameters of the discrete drum teeth on yarn quality, experiments were performed using the Full Factor Experiments mathematical planning method, and a regression equation was obtained for each variable. As a result, the optimal values of the discretization drum coating teeth of the OE spinning machine were determined.
\end{abstract}

\section{Introduction}

It is known that in order to increase the efficiency of separation of complex fibers into separate fibers and to straighten and parallel the ends of the fibers in the supplied sliver, it is recommended to increase the working speed of the discreting drum. To do this, it is advisable to use special tooth types so that there is a correlation between the parameters of the discrete drum teeth and the fiber properties. It has been found that the distance between the discrete drum teeth and the angle of inclination of the tooth have a large effect on the processing of the fiber bundle. To better separate the fibers into individual fibers, it is necessary to increase the angle of inclination of the anterior part of the dissecting drum teeth and reduce the area of impact of the tooth tip $[1,2]$.

Taking into account the above, in order to improve the yarn quality performance, theoretical and experimental studies have been conducted to produce a competitive product by changing the geometric shape of the discrete drum teeth and determining the optimal value of the angle of inclination of the tooth [3].

\section{Theoretical part}

To increase the intensity of the discreting drum (Fig. 1), it has been proved that increasing the number of teeth per $1 \mathrm{~cm}^{2}$ surface of the discretization drum can be done by applying the multi-input wrapping method of teeth and reducing the tooth pitch (Fig. 2) [4-6].

\footnotetext{
*Corresponding author: axmedov58@mail.ru
} 


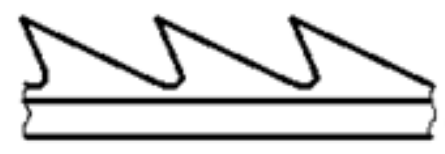

Fig. 1. Appearance of teeth wrapped around a discrete drum BD-330
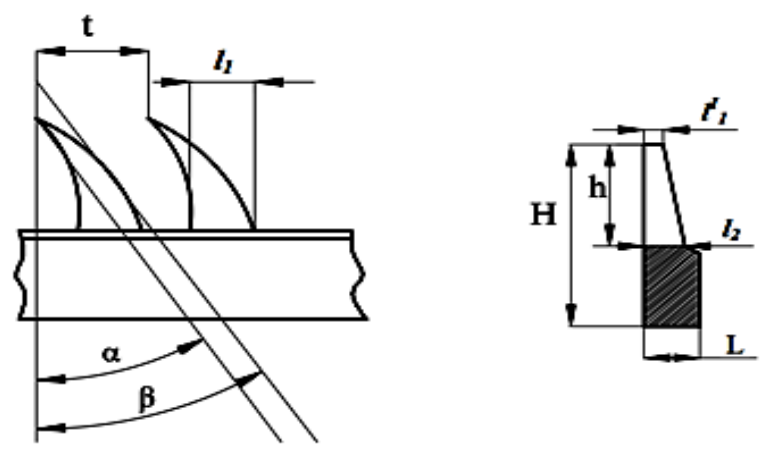

Fig. 2. A new construction tooth profile that wraps around the recommended discretion drum BD-330

In yarn formation, the flow of fiber to the continuous spinning chamber is done by discreting drum teeth. We analyze the motion of a massive fiber whose center is at point 0 in determining the motion along the base of the tooth, which varies along the surface of the discrete drum teeth (Fig. 3).

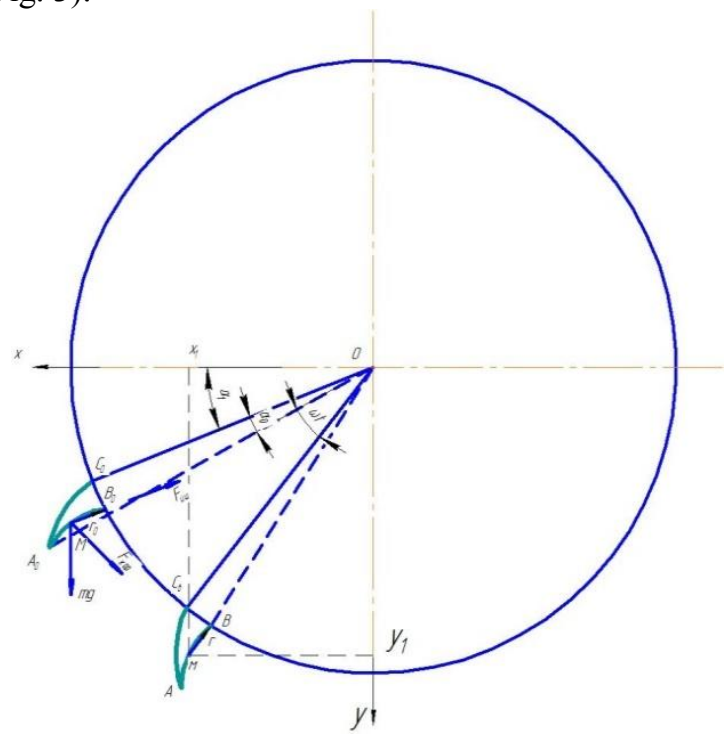

Fig. 3. Movement of fibers in discrete drum teeth.

Using the equation of motion of the fibers, we obtain the following equation. 


$$
\begin{aligned}
& x_{0}=R \cdot \cos \left(\alpha_{0}+\alpha_{1}\right)+r_{0} \cdot \sin \beta_{0} \\
& y_{0}=R \cdot \sin \left(\alpha_{0}+\alpha_{1}\right)+r_{0} \cdot \cos \beta_{0}
\end{aligned}
$$

Generalized coordinates are selected, which are the forces acting on the fibers. The coordinates of the kinetic energy of the fibers and the function of the velocities are determined (Fig. 4).

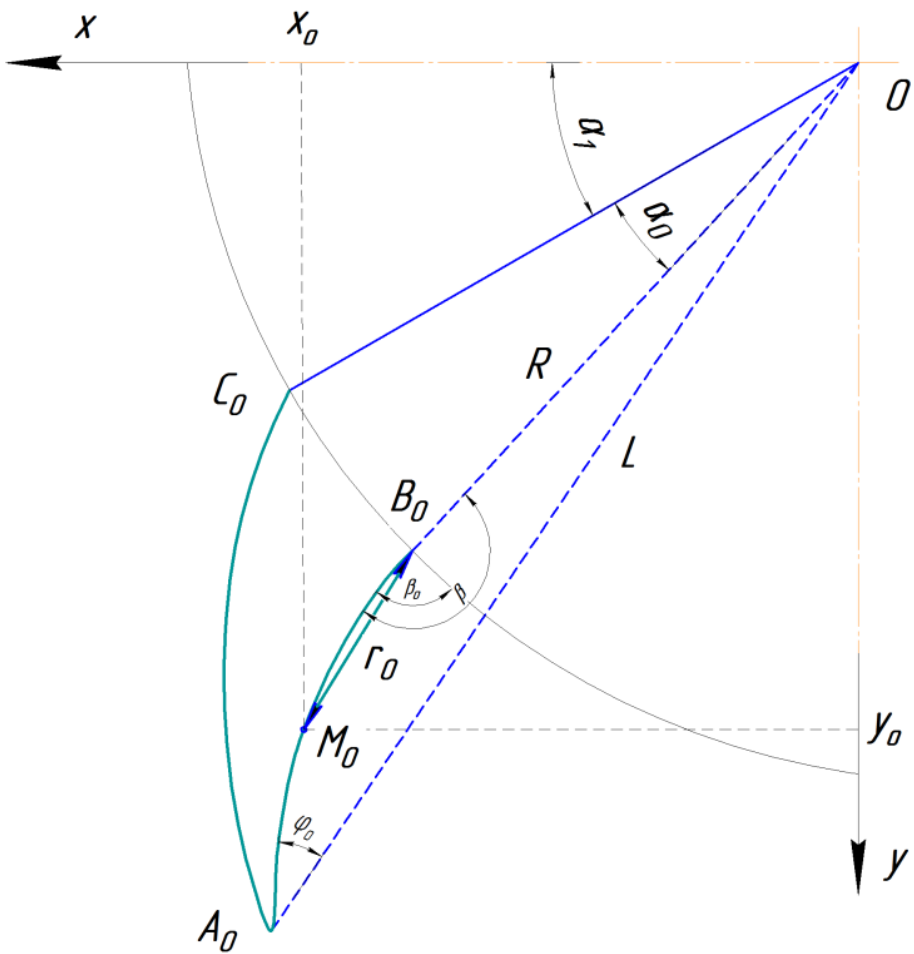

Fig. 4. The initial movement of the fibers at the base of the tooth
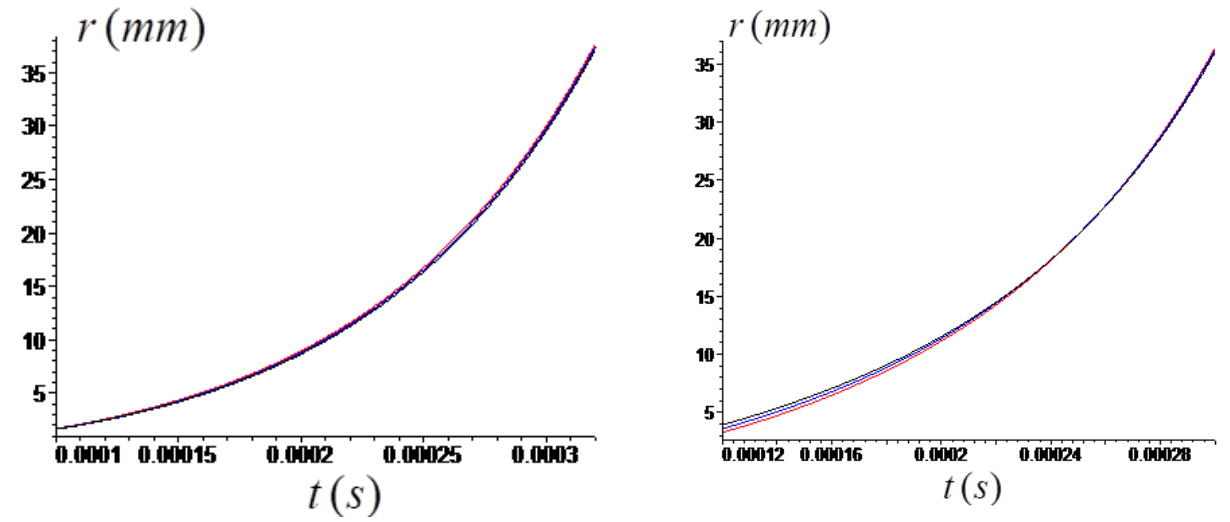

Fig. 5. Analysis of $\beta_{1}=64^{0} ; \beta_{2}=68^{0} ; \beta_{3}=72^{0}$ at different values of time and linear velocities of fiber motion and at different values of the motion of the fibers depending on the time and the angles of deflection 
Using the sine theorem, the following equation was derived:

$\frac{L}{\sin \beta}=\frac{R}{\sin \varphi_{0}}$ in this $\sin \beta=\frac{L}{R} \cdot \sin \varphi_{0}$ or $\beta=\arcsin \left[\frac{l}{R} \sin \varphi_{0}\right]$ at time $\mathrm{t}>0$. If the angle $\beta>0$ then it $\beta_{0}$ is as follows:

$$
\beta_{0}=\beta-\alpha_{0}-\alpha_{1}-\omega \cdot t
$$

In Fig. 5 analyzed for torsion angle values at $\beta_{1}=64^{0} ; \beta_{2}=68^{0} ; \beta_{3}=72^{0}$ graphs on the variation of the angular velocity of the fiber movement and the tilt angles are given.

The parameters affecting the cotton fiber of the newly recommended discrete drum teeth were analyzed (Fig. 6).

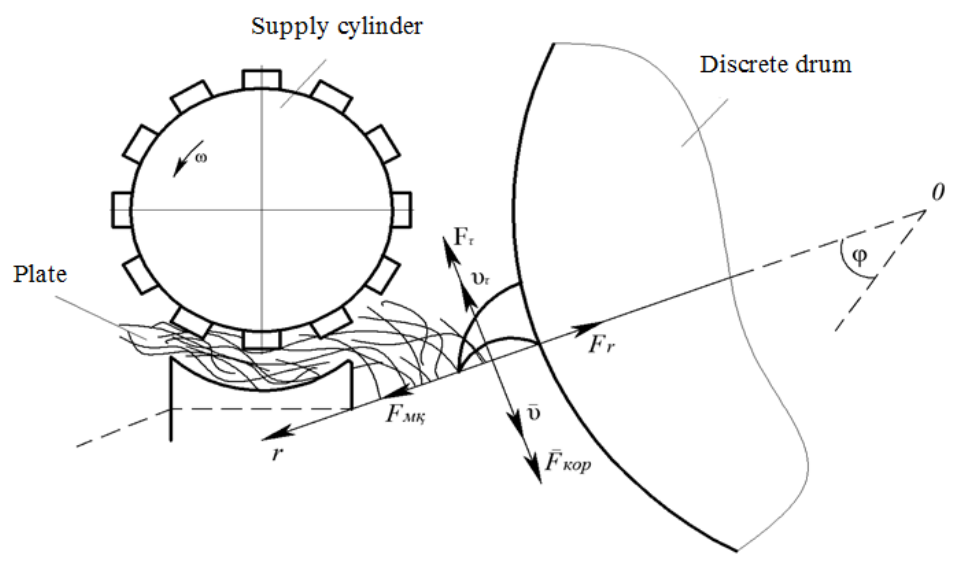

Fig. 6. BD-330 OE spinning machine supply zone diagram

If the rotational speed of the discrete drum is too high, the placement of the fibers in the transverse direction can affect the structure and quality of the yarn. Therefore, the rotational frequency of the discrete drum of the above OE spinning machine from 600 to $10000 \mathrm{rpm}$ values of the rotation frequency corresponding to the angle of inclination of the coating tooth was analyzed.

\section{Materials and Methods}

The aerodynamic drag forces acting on the fibers along the test and radius-vector were determined as follows.

$$
\begin{aligned}
& \vec{F}_{r}=\frac{1}{2} \cdot f_{r} \cdot S_{r} \cdot \rho \cdot \vartheta_{r}{ }^{2} \\
& \vec{F}_{\tau}=\frac{1}{2} \cdot f_{\tau} \cdot S_{\tau} \cdot \rho \cdot\left(\vartheta-\vartheta_{\tau}\right)^{2}
\end{aligned}
$$

The velocities of the fibers at the base of the discrete drum coating tooth along the shear and radius-vector were calculated (Table 1 and Fig. 7).

$$
\begin{aligned}
& \vartheta_{r}=\frac{d r}{d t}=\dot{r} \\
& \vartheta_{\tau}=r \cdot \frac{d \varphi}{d t}=r \cdot \dot{\varphi}
\end{aligned}
$$


Table 1. Values of variable parameters in determining the trajectory of fiber motion.

\begin{tabular}{|c|c|c|c|}
\hline Trajectory & $v\left(\mathrm{~min}^{-1}\right)$ & $\vartheta_{1}(m / s)$ & $\beta(\operatorname{deg}$ ree $)$ \\
\hline 1 & 6500 & 0.543 & 64 \\
\hline 2 & 6500 & 0.416 & 64 \\
\hline 3 & 6500 & 0.231 & 64 \\
\hline 4 & 7000 & 0.543 & 68 \\
\hline 5 & 7000 & 0.416 & 68 \\
\hline 6 & 7000 & 0.231 & 68 \\
\hline 7 & 7500 & 0.543 & 72 \\
\hline 8 & 7500 & 0.416 & 72 \\
\hline 9 & 7500 & 0.231 & 72 \\
\hline
\end{tabular}

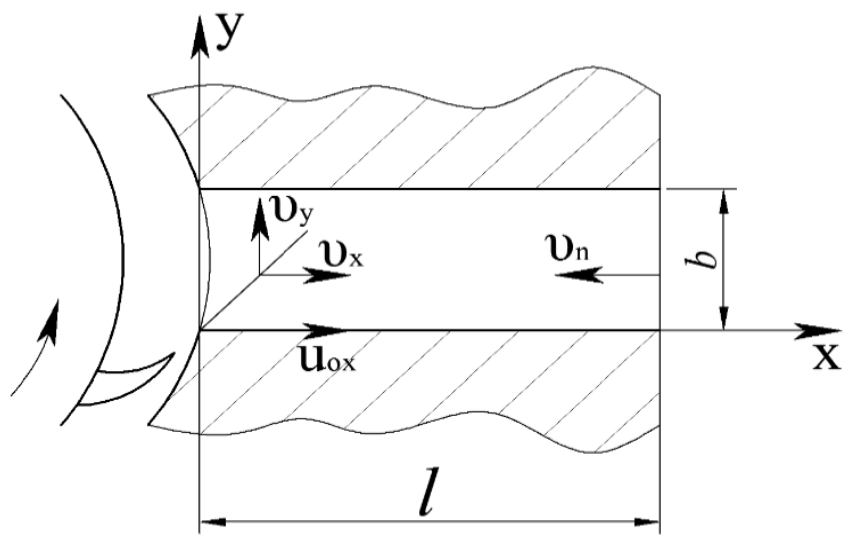

Fig. 7. Schematic diagram of small impurities and fibers in the channel for separating small particles and dust

Assuming that there is a constant cross-section of the channel for the separation of small particles and dust from the fibers, a coordinate system was chosen in which the $O X$ axis corresponds to the wall of the dust removal channel.

$$
\begin{aligned}
& m \cdot \frac{d \vartheta_{y}}{d t}= \pm \frac{1}{2} \cdot C_{y} \cdot S \cdot \rho \cdot \vartheta_{y}^{2} \\
& m \cdot \frac{d \vartheta_{x}}{d t}=-\frac{1}{2} \cdot C_{x} \cdot S \cdot \rho \cdot\left(\vartheta_{x}+\vartheta_{n}\right)^{2}
\end{aligned}
$$

Equation (4) represents the differential equations of motion along the channel of separation of fine particles and dust from fibers.

Here the fibers are the flowing surfaces $(S)$, the coefficients of resistance $C_{x}$ and $C_{y}$, the air density $(p)$, the mass of the fibers $(m)$.

$$
\begin{aligned}
& y=\frac{\vartheta_{t}^{2}}{g} \cdot \ln \left[\frac{g}{\vartheta_{t}^{2}}\left(t-t_{0}\right)+\frac{1}{k \vartheta_{y 0}}\right] \cdot k \vartheta_{y 0} \\
& x=-\vartheta_{n} t+\frac{\vartheta_{t}^{2}}{g} \cdot \ln \left[\frac{g}{\vartheta_{t}^{2}} t+\frac{1}{u_{0 x}+\vartheta_{n}}\right] \cdot k \vartheta_{y 0}
\end{aligned}
$$


Equations (5) show the movement of fibers along the separation channel of fine impurities and dust.

The equation of the total trajectory of the fibers along the channel was calculated:

$$
\left.l=\frac{\vartheta_{t}^{2}}{g} \cdot \ln \left[\ln \frac{u_{0 x}+\vartheta_{t}}{\vartheta_{n}}-\frac{u_{0 x}}{u_{0 x}+\vartheta_{n}}\right)\right] \cdot k \vartheta_{y 0}
$$

In order to separate fine impurities and dust from the fibers from the (6) equation of motion, the following condition must be met.

Thus, in the separation of fine impurities and dust from the fibers, the distance traveled by the fibers in the channel is determined by the value of the velocity component, as well as the magnitude and direction of the air flow velocity.

\section{Results and Discussion}

As the rotational frequency increases, the velocity of the fibers along the trajectory and the conditions of movement in the duct during fiber dust separation change with the direction of fiber movement and the reverse direction of air flow, the rotational frequency leads to changes in particulate matter and dust mass and quality composition (Fig. 8).
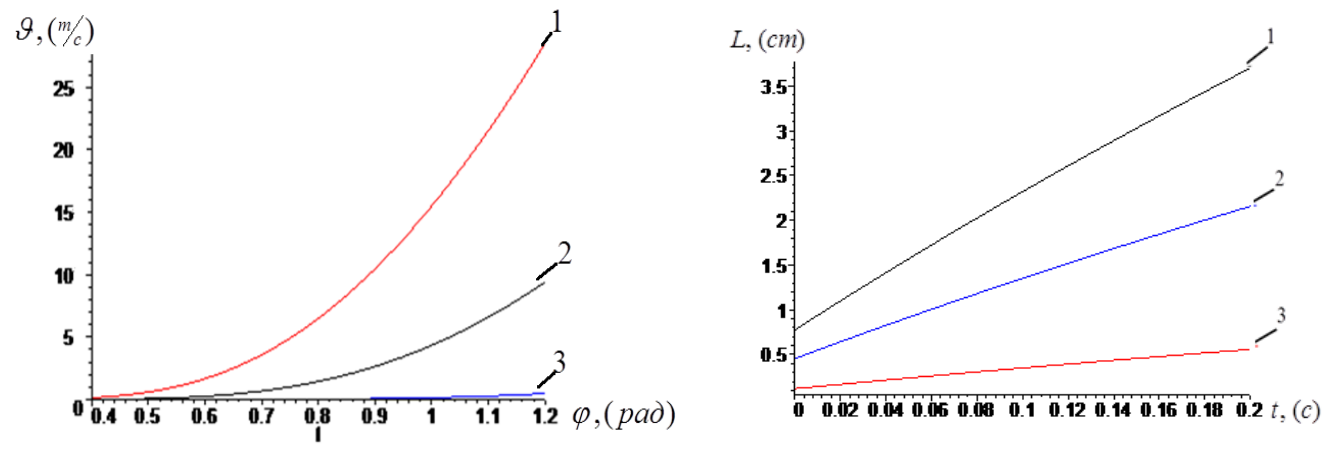

Fig. 8. Analysis of $\beta_{1}=64^{0} ; \beta_{2}=68^{0} ; \beta_{3}=72^{0}$ at different values of coating tooth deflection angles and different values of linear velocities depending on the time of movement under the tooth

The motion of the fibers under the influence of external forces was studied, and the angular velocity and deflection angles in it were calculated and graphs were given. Based on the analysis of the conditions of fiber retention with the discrete drum coating teeth, the equations for determining the angle of inclination of the teeth were obtained.

The equations of the change in the speed of the discrete drum, the force of gravity relative to the surface of the front ends of the tooth and the coefficient of friction of the centrifugal force, the mass of fibers held by the teeth were obtained [7].

An analytical expression of the law of motion of the fiber on the surface of the discrete drum teeth is given. As a result of the analysis of fiber tension and velocity from them, it was found that the tensile strength and velocity of the fiber increase with the increase of the discreting zone $[8,9]$.

The laws of fiber velocities, discrete drum teeth torsion angle, number of revolutions and time dependence are expressed in the form of graphs.

At the Department of "Spinning Technology" of the Tashkent Institute of Textile and Light Industry in the laboratory studied the effect of tooth pitch and the angle of inclination 
of the front edge of the tooth, as well as parameters of rotational frequency of the discrete drum on the quality of $\mathrm{OE}$ yarn $\mathrm{Ne} 24$ and stability of the spinning process.

Type 5, 1st grade, good grade, Bukhara-6 selection cotton fiber was used in the production of yarn. The Full Factor Experiments-23 method of mathematical planning was used in the calculations [8].

The following indicators were accepted as undesirable factors.

$x_{1}$ - disreting drum rotation frequency, $\min ^{-1}$;

$x_{2}$ - discreting drum tooth pitch, $\mathrm{mm}$;

$x_{3}$ - teeth front edge slope angle, grad.

In constructing regression equations, the coded values of the factors were coded in the range $(-1)$ to $(+1)$ for each independent variable. When the minimum value of the independent variable is given as "-1", and the maximum value in the range "+1", the corresponding coded values of the factors of the natural level are obtained.

The degree of variability of the factors is given in Table 1 and Table 2 in the experimental plan.

Table 1. Level of variability of factors

\begin{tabular}{|l|c|c|c|c|}
\hline \multirow{2}{*}{ Factors } & \multicolumn{3}{|c|}{ Factors level } & \multirow{2}{*}{$\begin{array}{c}\text { Variation } \\
\text { intervals }\end{array}$} \\
\cline { 2 - 4 } & - & $\mathbf{0}$ & + & 500 \\
\hline $\begin{array}{l}x_{1}-\text { disreting drum rotation frequency, } \\
\mathrm{min}^{-1}\end{array}$ & 6500 & 7000 & 7500 & 1,0 \\
\hline$x_{2}-$ discreting drum tooth pitch, $\mathrm{mm}$ & 2,5 & 3,5 & 4,5 & 4 \\
\hline$x_{3}$ - teeth front edge slope angle, grad & 64 & 68 & 72 & 4 \\
\hline
\end{tabular}

Optimization parameters:

$\mathrm{y} 1$ - tensile strength of the yarn, $\mathrm{cm} / \mathrm{tex}$

y2 - CV in breaking strength, \%

y3 - yarn appearance defects, $\mathrm{km}$

Regression equations were constructed for each optimization parameter.

Evaluation of the values of the coefficients of regression equations is carried out by the Student's criterion, the assessment of the adequacy (suitability) of the equation is carried out by the Fisher criterion $[8,9]$.

Table 2. Experimental plan and results (on the specific tensile strength of the yarn)

\begin{tabular}{|c|c|c|c|c|c|c|c|c|c|c|}
\hline & \multicolumn{3}{|c|}{ Factors } & \multicolumn{7}{|c|}{ Experiments } \\
\cline { 2 - 11 } & $\mathbf{X}_{\mathbf{1}}$ & $\mathbf{X}_{\mathbf{2}}$ & $\mathbf{X}_{\mathbf{3}}$ & $y_{i 1}$ & $y_{i 2}$ & $y_{i 3}$ & $\bar{y}_{u}$ & $S_{u}^{2}$ & $\hat{y}_{u}$ & $R_{0}(\%)$ \\
\hline 1 & - & - & - & 11.4 & 10.6 & 11.3 & 11.1 & 0.57 & 11.815 & 0.69 \\
\hline 2 & + & - & - & 10.6 & 9.8 & 10.8 & 10.4 & 0.68 & 11.086 & 0.40 \\
\hline 3 & - & + & - & 11.02 & 10.85 & 11.01 & 10.96 & 0.48 & 11.432 & 0.25 \\
\hline 4 & + & + & - & 11.8 & 10.7 & 11.10 & 11.5 & 0.43 & 11.043 & 0.93 \\
\hline 5 & - & - & + & 11.9 & 11.2 & 11.1 & 11.4 & 0.46 & 12.21 & 0.93 \\
\hline 6 & + & - & + & 10.6 & 10.7 & 10.1 & 10.8 & 0.62 & 10.96 & 0.25 \\
\hline 7 & - & + & + & 11.9 & 11.4 & 11.8 & 11.7 & 0.37 & 11.42 & 0.40 \\
\hline 8 & + & + & + & 11.7 & 12.01 & 11.84 & 11.85 & 0.40 & 10.93 & 0.69 \\
\hline
\end{tabular}


Evaluation of the quality of the raw product and yarn is tested on modern laboratory equipment (Table 3 ).

Table 3. Table summarizing the results of experiments

\begin{tabular}{|c|c|c|c|c|c|c|c|c|c|c|c|c|}
\hline \multirow{3}{*}{ \# } & \multicolumn{7}{|c|}{ Coding the value of factors } & \multicolumn{4}{|c|}{ Optimization parameters } & \multirow[b]{2}{*}{ 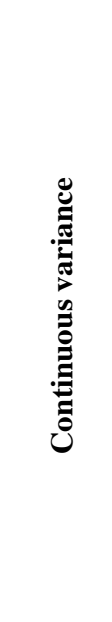 } \\
\hline & \multirow[t]{2}{*}{$\mathbf{x}_{1}$} & \multirow[t]{2}{*}{$\mathbf{x}_{2}$} & \multirow[t]{2}{*}{$\mathbf{x}_{3}$} & \multirow[t]{2}{*}{$\stackrel{a}{a}$} & \multirow[t]{2}{*}{$\stackrel{\ddot{A}}{\ddot{A}}$} & \multirow[t]{2}{*}{$\underset{\ddot{z}}{\ddot{*}}$} & \multirow[t]{2}{*}{ ه্d } & 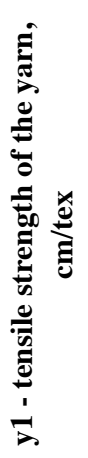 & 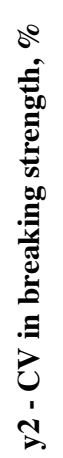 & 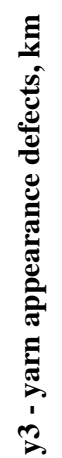 & 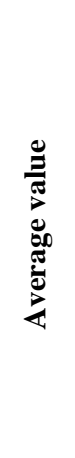 & \\
\hline & & & & & & & & $\bar{Y}_{1}$ & $\bar{Y}_{2}$ & $\bar{Y}_{3}$ & $\bar{Y}$ & $S^{2}(Y)$ \\
\hline 1 & - & - & - & + & + & + & - & 11.1 & 9.4 & 141 & 53.8 & 5699.3 \\
\hline 2 & + & - & - & - & - & + & + & 10.4 & 9.7 & 153 & 57.7 & 6811.7 \\
\hline 3 & - & + & + & - & + & - & + & 10.96 & 9.5 & 130 & 50.2 & 4782.2 \\
\hline 4 & + & + & - & + & - & - & - & 11.5 & 9.1 & 124 & 48.2 & 43106.7 \\
\hline 5 & - & - & + & + & - & - & + & 11.4 & 9.3 & 128 & 49.6 & 4615 \\
\hline 6 & + & - & + & - & + & - & - & 10.8 & 9.6 & 146 & 55.5 & 6147.7 \\
\hline 7 & - & + & + & - & - & + & - & 11.7 & 8.8 & 116 & 45.5 & 6294.8 \\
\hline 8 & + & + & + & + & + & + & + & 11.85 & 8.6 & 120 & 46.8 & 4075 \\
\hline
\end{tabular}

The model has a second-order polynomial shape:

$$
y=b_{o}+\sum_{i=1}^{N} b_{i} x_{i}+\sum_{i j=1}^{N} b_{i j} x_{i} x_{j}+\sum_{i=1}^{N} b_{i i} x_{i}^{2}
$$

where, $b_{0}, b_{i}, b_{i j}$ - the estimates of the model coefficients; $N$ - number of experiments; and, $\mathrm{x}_{\mathrm{i}}, \mathrm{x}_{\mathrm{j}}$ - are factors.

After subtracting the insignificant coefficients, the regression equations look like this:

$$
\begin{gathered}
y_{1}=11,26+0,157 x_{1}-0,448 x_{2}-0,2 x_{3}+0,35 x_{1} \cdot x_{3}-0,2 x_{2} \cdot x_{3} \\
y_{2}=9,21-0,117 x_{1}+0,383 x_{2}-0,325 x_{1} \cdot x_{3}+0,1 x_{2} \cdot x_{3} \\
y_{3}=132,88-5,5 x_{1}+8,33 x_{2}+8,25 x_{1} \cdot x_{2}-8,25 x_{1} \cdot x_{3}+8,5 x_{2} \cdot x_{3}
\end{gathered}
$$


To evaluate the adequacy of equations (8-10), the calculated values $y_{1}, y_{2}, y_{3}$ were determined (Table 3 ). The adequacy of the equations (8-10) was evaluated using the F-ratio (Fisher's criterion) [9]:

$$
F=s_{a g i}^{2} / s_{y i}^{2}
$$

Where, $s_{a g i}^{2}$-equality variance is calculated according to Equation (11)

The exact values of the $y_{\mathrm{r}}$ values calculated as the adequacy variance are a measure of the deviation from $y_{f}$.

\section{Conclusions}

Thus, from the compatibility of Equations 8-10, it can be concluded that:

- the tensile strength of the yarn increases with increasing rotational frequency of the discreting drum and decreasing the pitch of the tooth. Increasing the angle of inclination of the tooth is advisable when the tooth pitch is not large.

- the $\mathrm{CV}$ in the breaking force decreases with increasing discretion drum rotation frequency, decreasing tooth pitch and increasing the front edge slope angle;

- the total amount of appearance defects decreases with increasing discretion drum rotation frequency and increases sharply with increasing tooth pitch, increasing the front edge slope angle reduces the number of yarn imperfection, but their amount increases sharply with increasing tooth pitch.

Using the obtained regression dependencies, the following optimization task was constructed: minimizing the amount of appearance defects as a result of high limits of specific breaking strength and low limits of $\mathrm{CV}$ in breaking strength. Option 8 is suitable for this optimization task. The rotational frequency of the discrete drum is $7000 \mathrm{rpm}$, the pitch of the tooth is $2.5 \mathrm{~mm}$, and the angle of inclination of the front edge of the teeth is 680.

Proper selection of the parameters of the sampling drum increases the stability of the OE spinning process. Yarn breaks decreased from 38 to 26 breaks per 1,000 chambers.

\section{References}

1. V. E. Ribin, Fiber 17(13), 17 (2005)

2. S. N. Khripunov, Fiber 9, 11 (2000)

3. A. Pirmatov, Fiber technology, 149 ("Ijod Print" Press, Tashkent, 2020)

4. YU. V. Pavlov, Technology of cotton fiber, 521 (Ivanovo Press, Moscow, 2000)

5. F. M. Plekhanov, Theory of fibering, 298 (Nauka Press, Moscow, 2000)

6. A. R. Horrocks, S. Anand, Handbook of technical textiles, 576 (The Bolton Institute Press, UK, 2000)

7. T. R. Rashidov, SH. SHoziyotov, Q. B. Mo“minov, Theoretical Mechanics 290-295 (1990)

8. M. M. Varkovetskiy, Light Industry, $72-73$ (1982)

9. A. G. Sevostyanov, Light Industry 12, 165-167 (2007) 